\title{
Non-Linear Excitation of Warps by Spiral Waves in Galaxies
}

\author{
F. Masset and M. Tagger \\ Service d'Astrophysique, CE-Saclay, F-91191 Gif/Yvette Cedex, France
}

\begin{abstract}
.
We present a mechanism to explain the permanent excitation of warps in spiral or barred galaxies. We show that non-linear mode coupling with the spiral wave can be very efficient to drive two warp waves. We derive an expression of the coupling coefficient in the WKB approximation. We show that the coupling is too weak in the stellar disk, except at the Outer Lindblad Resonance where the spiral slows down and is efficiently coupled to warp waves. There, the spiral can almost completely transfer its energy and angular momentum to a "transmitted" warp, which we can observe in HI, and a "reflected" one, which we can observe as a corrugation. Our mechanism reproduces the observed amplitudes of the warp and of the corrugation.

We first present side results concerning the propagation of warps in moderately thick disks.
\end{abstract}

\section{Linear results: Propagation of Warps in Moderately Thick Disks}

In an infinitely thin disk, the dispersion relation of bending waves is: $\tilde{\omega}^{2}=$ $\mu^{2}+2 \pi G \Sigma k$, where $\tilde{\omega}$ is the warp's frequency in the frame of matter (if $\omega$ is its frequency in the galactocentric frame, $\tilde{\omega}=\omega-m \Omega$, where $m=1$ for the typical "integral sign" shaped warps and $\Omega$ is the orbital frequency), $\Sigma$ is the surface density of the galactic disk, and $k$ is the wavevector modulus of the warp wave.

We have derived a new dispersion relation taking into account the finite disk thickness and compressibility for an isothermal and gaseous disk, with sound speed $a$. We have performed the calculations under two classical assumptions:

- The WKB assumption which implies that the wave is tightly wound and that the relative variation of its radial wavelength over one period is small (i.e. $\partial \ln k / \partial r \ll k$ ).

- The shearing sheet approximation, which consists in rectifying a narrow annulus around the corotation of the warp.

A last assumption is that the warp wavelength is greater than the disk thickness. This assumption is true for the external bending of the HI layer of the galactic disk as well as for shorter wavelength corrugation waves of the internal gaseous layer.

We have obtained (Masset \& Tagger 1995): 


$$
\tilde{\omega}^{2}=\mu^{2}+2 \pi G \Sigma k+a^{2} k^{2} \frac{\tilde{\omega}^{2}}{\tilde{\omega}^{2}-\kappa^{2}}
$$

where $\kappa$ is the epicyclic frequency. This dispersion relation corresponds to the classical one with an additional term obviously related to horizontal motions (through the epicyclic frequency $\kappa$ ) and to compressional effects (through the sound speed $a$ ). In fact, horizontal motions are generated by horizontal pressure gradients which arise due to the vertical stratification of pressure and to the bending of the disk by the warp.

\section{Non-Linear Excitation of Warps}

\subsection{Introduction}

We consider non-linear coupling between a spiral wave and two warp waves. The second order terms in the perturbed quantities are almost everywhere negligible compared to first order ones, except at some well defined positions in the disk, where the coupling efficiency can be high enough to make second order terms comparable to linear ones. At these positions (which we will identify as Lindblad or vertical resonances), the coupling of the three waves may lead to an efficient energy and momentum transfer between the spiral density wave and the two bending waves. Non-linear coupling of spiral waves has already given convincing explanations of observations and numerical simulations (Tagger et al. 1987 and Sygnet et al. 1988).

\subsection{Notion of Mode Coupling}

We write hydrodynamic equations (continuity and Euler equations) to second order in the perturbed quantities, and we project these equations on the azimuthal wavenumber and pulsation of one of the bending waves (hereafter called warp 1). Provided the three waves obey some selection rules, the cross-terms will contribute to these projections. The selection rules required are: $m_{1}+m_{2}=m_{S}$ and $\omega_{1}+\omega_{2}=\omega_{S}$, where $S$ is for the spiral and 1 and 2 for both bending waves. It is worth mentioning that we do not consider any selection rule on the radial wavenumber $k$ since we expect the coupling to act only locally, in an annular region whose radial extent is small compared to the typical wavelength of the three waves involved.

An additional selection rule on the $z$-parity of the waves involved is required. Here we consider two warps, which have an odd $z$-parity. Their cross term will be even in $z$, as is the parity of a spiral density wave.

\subsection{Evaluation of the Coupling Efficiency}

The coupling efficiency is estimated analytically from the hydrodynamic equations. We use variational forms to write it as an exchange of energy and angular momentum between the spiral and the warps. For the sake of simplicity, we restrict ourselves to a search of a stationary solution, so that the energy transfer appears only as a radial variation of the energy flux of each wave (which would 


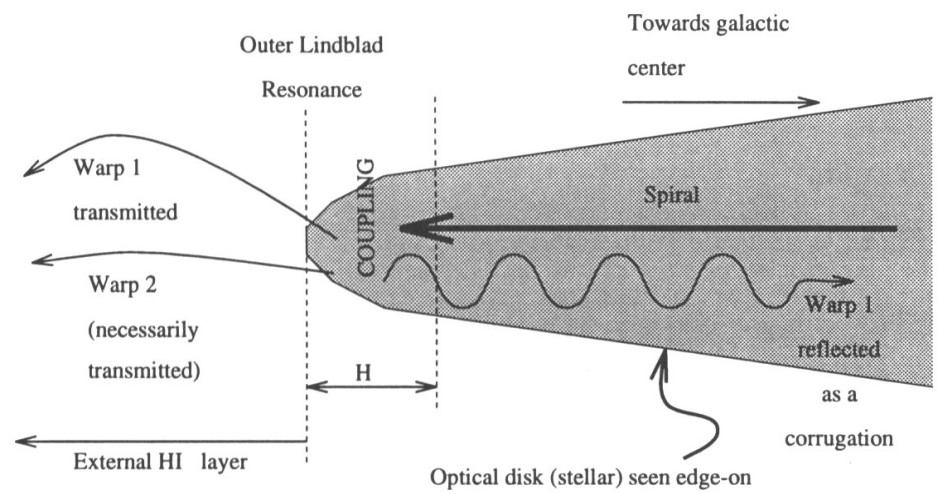

Figure 1. A schematic of the formation of warps

be constant in linear theory). For warp 1, the coupling equation can be written as:

$$
\partial\left(c_{g_{1}} E_{1}\right) / \partial r=\tilde{\omega}_{1} \nu_{Z}^{2} Z_{1} Z_{2} \sigma
$$

where $c_{g_{1}}$ is the group velocity of warp $1, E_{1}$ its density energy, $\nu_{Z}$ a characteristic vertical oscillation frequency, $Z_{1}$ and $Z_{2}$ the amplitude of both warps and $\sigma$ the perturbed surface density of the spiral. Similar equations can be written for the two other waves.

It is an easy matter to check that this set of equations will lead to a powerful coupling if the group velocities vanish at the same place. In fact, the group velocities vanish close to the resonances (Lindblad or vertical) of the waves. Now it is not possible that three waves which fulfill the selection rules have their resonances at the same radius. The best that can be achieved is that the vertical resonance of one bending wave coincides with the Lindblad resonance of the spiral. With such a set of waves, we have shown that the coupling efficiency is large enough to transfer nearly all the energy of the spiral wave to the two warp waves. The predictions of this mechanism agree with the observations, for the position at which the warp appears (at the optical edge, i.e. near the spiral OLR) as well as for the expected amplitude of both warps. Note that one of the warps, which is close to its forbidden band, can propagate only in one direction (outward), while the other one propagates both inward and outward. The inward propagating wave can be identified as a corrugation wave. The situation is depicted on the Figure 1.

\section{References}

Masset, F. \& Tagger, M. 1995, A\&A, in press

Sygnet, J. F., Tagger, M., Athanassoula, E., \& Pellat, R. 1988, MNRAS, 232, 733

Tagger, M., Sygnet, J.F., Athanassoula, E., \& Pellat, R. 1987, ApJ, 318, L43 